\title{
APPROXIMATIONS AND FIXED POINTS FOR CONDENSING NON-SELF-MAPS DEFINED ON A SPHERE
}

\author{
TZU-CHU LIN
}

(Communicated by Paul S. Muhly)

\begin{abstract}
In this paper, we investigate the validity of an interesting theorem of Ky Fan [Theorem 2, Math. Z. 112 (1969), 234-240] defined on a sphere (the boundary of a closed ball) in an infinite-dimensional Banach space. We will prove that it is true for a continuous condensing map with suitable conditions posed. As applications of our theorem, some fixed point theorems of continuous condensing non-self-maps defined on a sphere are derived. Our results generalize some results of R. Nussbaum [10] and P. Massatt [8].
\end{abstract}

Most fixed point theorems in Banach spaces deal with some classes of maps defined on a compact convex (or star-shaped) subset, a closed bound convex (or star-shaped) subset, or a closed bound subset with nonempty interior. What about the domain of a function which is neither of the above cases? The simplest interesting case would be a sphere (the boundary of a closed ball). It is clear that a continuous self-map defined on a sphere may not have fixed points; for example, a rotation of a sphere (or circle) in a plane. Nussbaum [10] proved that a continuous $k$-set-contractive map from a sphere into a sphere has a fixed point, if the dimension of the Banach space is infinite. Recently, Massatt [8] generalized this result to continuous condensing maps. For definitions of $k$-setcontractive and condensing maps, see for example [9 or 7$]$. Generalizations of fixed point theory from self-maps to non-self-maps has been a very active topic in nonlinear functional analysis in the past two decades. Does a continuous condensing non-self-map defined on a sphere have a fixed point? Under which conditions?

On the other hand, Fan [3] proved the following interesting theorem:

Let $K$ be a nonempty compact convex subset of a normed linear space $X$. Let $f$ be a continuous map from $K$ into $X$; then there exists a point $u$ in $K$ such that $\|u-f(u)\|=d(f(u), K)$.

The author [5] proved that it is true for a continuous condensing map defined on a closed ball of a Banach space. Is this still true for a continuous condensing map defined on a sphere of an infinite-dimensional Banach space? Under which conditions?

Received by the editors March 23, 1987.

1980 Mathematics Subject Classification (1985 Revision). Primary 47H10; Secondary 41A50. 
In this paper, we will prove that the second question is true under appropriate conditions (Theorem 1 below). As applications of our theorem, we derive fixed point theorems for continuous condensing non-self-maps defined on a sphere under suitable conditions, which answer the first question and also generalize Massatt's result [8].

We also remark that for a continuous condensing (even more generally, 1-setcontractive) map defined on a closed bounded convex subset of a Hilbert space, the above result of Fan is still true; see the author and Yen [5-7].

Throughout this paper, we will denote

$$
\begin{aligned}
& S_{r}=\{x \in X \mid\|x\|=r\}, \quad B_{r}=\{x \in X \mid\|x\|<r\}, \\
& I_{A}(x)=\{x+c(z-x) \mid \text { for some } z \in A, \text { some } c>0\},
\end{aligned}
$$

$\bar{D}$ the closure of $D$, where $X$ is a Banach space, $r$ a positive number, $A$ a convex set in $X$ and $D$ a set in $X$. It is clear that $A \subset I_{A}(x)$.

Now, we prove our main theorems.

Theorem 1. Let $S_{r}$ be a sphere with center at the origin and radius $r$ in an infinite-dimensional Banach space $X$. Let $f$ be a continuous condensing map from $S$ into $X$. If

$$
\|f(x)\| \geq r \text { for each } x \in S_{r},
$$

then there exists a point $u \in S_{r}$ such that

$$
\|u-f(u)\|=d\left(f(u), S_{r}\right)=d\left(f(u), \bar{B}_{r}\right) .
$$

Proof. Define

$$
R(x)= \begin{cases}x, & \text { if }\|x\| \leq r, \\ r x /\|x\|, & \text { if }\|x\| \geq r .\end{cases}
$$

From Nussbaum [9, Corollary 1], $R$ is a continuous 1-set-contractive map from $X$ onto $\bar{B}_{r}$. Let $F(x)=R \circ f(x)$, then $F$ is also a continuous condensing map. Since $\|f(x)\| \geq r$ for each $x \in S_{r}$, we have

$$
\|F(x)\|=\left\|\frac{r f(x)}{\|f(x)\|}\right\|=r,
$$

this implies $F(x) \in S_{r}$ and $F: S_{r} \rightarrow S_{r}$. From Massatt [8], $F$ has a fixed point in $S_{r}$, say $u$. Therefore

$$
\begin{aligned}
\|u-f(u)\| & =\|F(u)-f(u)\|=\|R(f(u))-f(u)\| \\
& =\left\|\frac{r f(u)}{\|f(u)\|}-f(u)\right\|=\|f(u)\|-r .
\end{aligned}
$$

For any $x \in S_{r}$ or $x \in \overline{B_{r}}$, we have

$$
\|f(u)\|-r \leq\|f(u)\|-\|x\| \leq\|f(u)-x\| .
$$

Hence

$$
\|u-f(u)\|=d\left(f(u), S_{r}\right)=d\left(f(u), \overline{B_{r}}\right) .
$$


Theorem 2. Let $S_{r}, B_{r}, f$, and $X$ be defined as in Theorem 1. Moreover, let $f$ satisfy any one of the following conditions:

(i) For each $x \in S_{r}$, with $x \neq f(x)$, there exists $y$ in $I_{\bar{B}_{r}}(x)$ such that

$$
\|y-f(x)\|<\|x-f(x)\| .
$$

(ii) $f$ is weakly inward (i.e., $f(x) \in \overline{I_{\overline{B_{r}}}(x)}$ for each $x \in S_{r}$ ).

(iii) $\|f(x)-x\|^{2} \geq\|f(x)\|^{2}-r^{2}$, for each $x \in S_{r}$.

Then $f$ has a fixed point in $S_{r}$.

Proof. From Theorem 1, there exists a point $u \in S_{r}$ such that

$$
\|u-f(u)\|=d\left(f(u), S_{r}\right)=d\left(f(u), \overline{B_{r}}\right) .
$$

If $f$ satisfies (i), and $u \neq f(u)$, then there exists $y$ in $I_{\bar{B}_{r}}(u)$ such that $\|y-f(u)\|<\|u-f(u)\|$. Since $y \in I_{\bar{B}_{r}}(u)$, there exists $z \in \bar{B}_{r}, c>0$ such that $y=u+c(z-u)$. Actually, $c>1$, otherwise $y \in \overline{B_{r}}$ which contradicts (1). Since

$$
z=u+\frac{1}{c}(y-u)=\left(1-\frac{1}{c}\right) u+\frac{1}{c} y=(1-\beta) u+\beta y,
$$

where $0<\beta=1 / c<1$, we have

$$
\begin{aligned}
\|z-f(u)\| & \leq(1-\beta)\|u-f(u)\|+\beta\|y-f(u)\| \\
& <(1-\beta)\|u-f(u)\|+\beta\|u-f(u)\| \\
& =\|u-f(u)\| .
\end{aligned}
$$

which contradicts (1). Therefore $u=f(u)$. It is clear that if $f$ satisfies (ii), then $f$ satisfies (i). If $f$ satisfies (iii), we will show that $u$ is a fixed point of $f$. From (iii),

$$
\|f(u)-u\|^{2} \geq\|f(u)\|^{2}-r^{2} .
$$

Since $\|f(u)\| \geq r$, we have $f(u) \neq 0$ and $r f(u) /\|f(u)\| \in S_{r}$. Therefore, from (1),

and

$$
\|u-f(u)\| \leq\left\|\frac{r f(u)}{\|f(u)\|}-f(u)\right\|=\|f(u)\|-r,
$$

$$
\|u-f(u)\|^{2} \leq(\|f(u)\|-r)^{2} .
$$

Combining (2) and (3), we have $\|f(u)\| \leq r$ and hence $\|f(u)\|=r$. From (1), $u$ is a fixed point of $f$.

Remark. Condition (i) of Theorem 2 was first stated by Browder [2]. Condition (ii) was first considered by Halpern [4], and (iii) was considered by Altman [1]. To the best of my knowledge, Theorem 2 has not been derived through other approaches. In other words, even Theorem 2 is new to the mathematical literature. We also note that Massatt's result [8] only appeared recently, in 1983. 
Corollary (MASsatt [8]). Let $X, S_{r}$ be defined as in Theorem 1 , and let $f$ be a continuous condensing map from $S_{r}$ into $S_{r}$. Then $f$ has a fixed point.

Proof. Since $f(x) \in S_{r} \subset \overline{B_{r}} \subset I_{\bar{B}_{r}}(x)$, from Theorem 2(ii), $f$ has a fixed point.

Finally, we give an example of a continuous condensing map $f$, with $\|f(x)\|<r$, such that the conclusion of Theorem 1 is still true, but $f$ has no fixed point in $S_{r}$.

Example. Let $S_{r}$ be a sphere in a Banach space $X$ (finite-dimensional or infinite-dimensional). Let $f: S_{r} \rightarrow X$ and $f(x)=x / 2$ for each $x \in S_{r}$. Then $f$ is a contraction and is a continuous condensing map. Clearly $f$ has no fixed point in $S_{r}$. But for every $u \in S_{r}$, we have

$$
\|u-f(u)\|=d\left(f(u), S_{r}\right) .
$$

In fact, $\|u-f(u)\|=\|u\| / 2=r / 2$ and

$$
\|x-f(u)\|=\| x-u / 2|| \geq|||x||-||u / 2|||=|r-r / 2|=r / 2,
$$

for $x \in S_{r}$. Therefore $\|u-f(u)\|=d\left(f(u), S_{r}\right)$. Certainly, in this example,

$$
d\left(f(u), S_{r}\right) \neq d\left(f(u), \overline{B_{r}}\right) \text {. }
$$

\section{REFERENCES}

1. M. Altman, A fixed point theorem in Hilbert space, Bull. Polish Acad. Sci. 5 (1957), 19-22.

2. F. E. Browder, On a sharpened form of the Schauder fixed point theorem, Proc. Nat. Acad. Sci. U.S.A. 74 (1977), 4749-4751.

3. K. Fan, Extensions of two fixed point theorems of F. E. Browder, Math. Z. 112 (1969), 234-240.

4. B. Halpern, Fixed point theorems for set-valued maps in infinite dimensional spaces, Math. Ann. 189 (1970), 87-98.

5. T. C. Lin, A note on a theorem of Ky Fan, Canad. Math. Bull. 22 (1979), 513-515.

6. T. C. Lin and C. L. Yen, Applications of the proximity map to fixed point theory, Approximation Theory and Applications (S. P. Singh, ed.), Research Notes in Math., vol. 133, Pitman, 1985, pp. 96-103.

7. T. C. Lin and C. L. Yen, Applications of the proximity map to fixed point theorems in Hilbert space, J. Approx. Theory 52 (1988), 141-148.

8. P. Massatt, $A$ fixed point theorem for $\alpha$-condensing maps on a sphere, Proc. Roy. Soc. Edinburgh Sect. A 94 (1983), 323-329.

9. R. D. Nussbaum, The fixed point index for local condensing maps, Ann. Mat. Pura Appl. 89 (1971), 217-258.

10. R. D. Nussbaum, Some fixed point theorems, Bull. Amer. Math. Soc. 77 (1971), 360-365.

Department of Mathematics, University of Wisconsin, Milwaukee, Wisconsin 53201

Current address: Department of Applied Mathematics, National Chiao Tung University, Hsinchu, Taiwan, R.O.C. 